\title{
ANÁLISIS DEL RIESGO POTENCIAL AMBIENTAL POR ALQUILFENOLES PRESENTES EN AGUAS DEL RÍO CAUCA A SU PASO POR LA ZONA URBANA DE CALI (VALLE DEL CAUCA, COLOMBIA)*
}

\author{
Gloria Amparo Jiménez-Botero ${ }^{1}$, Alejandro Soto-Duque ${ }^{2}$, Ricardo Álvarez-León ${ }^{3}$
}

\begin{abstract}
Resumen
Objetivos: Analizar el riesgo ambiental en cinco estaciones de muestreo sobre el río Cauca, debido a la presencia de fenoles, detectada previamente, especialmente teniendo como base que algunos de los alquilfenoles encontrados presentan riesgo de disrupción endocrina en mamíferos incluyendo el hombre. Alcance: Inventario químico cualitativo de las aguas colectadas y analizadas. Metodología: Se aplicó la técnica de cromatografía de gases acoplada a espectrometría de masas, pudiendo identificar su riesgo, con el soporte de las bases de datos: Pubmed Compounds, Pubmed Chem, Science Direct, IARC -International Agency of Research on Cancer- y las hojas de seguridad -MSDS-. Principales resultados: Se identificaron 70 compuestos orgánicos, entre los cuales están algunos alquilfenoles, de los cuales presentan riesgo a la salud humana y a los ecosistemas acuáticos, otros se encuentran sin caracterización del riesgo, y un último grupo sin información. Conclusiones: Es un trabajo pionero para entender los riesgos de la contaminación urbana, agrícola e industrial del río, a su paso por la ciudad de Cali, y será de gran utilidad para que los entes de control de la región, como la Corporación Autónoma Regional del Valle del Cauca, CVC, puedan tomar las medidas necesarias para la gestión adecuada del río.
\end{abstract}

Palabras clave: contaminación, cromatografía, alquilfenoles, riesgos ambientales, río Cauca, Colombia.

\footnotetext{
*FR: 11-VII-2014 . FA: .4-III-2015

${ }^{1}$ Magister. Docente investigadora grupo GEADES. Universidad Autónoma de Occidente. Cali (Valle) Colombia. E-mail: gajimenez@uao.edu.co

${ }^{2}$ Magister. Docente investigador grupo GEADES. Universidad Autónoma de Occidente. Cali (Valle) Colombia. E-mail: asoto@uao.edu.co

${ }^{3}$ Magister. Docente investigador. Universidad de Manizales. Manizales (Caldas) Colombia. E-mail: ricardoalvarezleon@gmail.com
}

JIMÉNEZ-BOTERO, G.A., SOTO-DUQUE, A. \& ÁLVAREZ-LEÓN, R., 2015.- Análisis del riesgo potencial ambiental por alquilfenoles presentes en aguas del río Cauca a su paso por la zona urbana de Cali (Valle del Cauca, Colombia). Bol. Cient. Mus. Hist. Nat. U. de Caldas, 19 (1): 43-48. DOI: 10.17151/bccm.2015.19.1.3 


\title{
POTENTIAL ENVIRONMENTAL RISK ANALYSIS FOR ALKYL PHENOLS PRESENT IN RIVER WATERS CAUCA PASSING THROUGH THE URBAN AREA OF CALI (VALLE DEL CAUCA, COLOMBIA)
}

\begin{abstract}
Objectives: Analyzing environmental risk five sampling stations on the Cauca, due to the presence of phenol, previously detected, especially on the basis of some of the alkyl phenols found at risk of endocrine disruption in mammals including man. Scope: Qualitative chemical inventory of water collected and analyzed. Methodology: The technique of gas chromatography coupled to mass spectrometry was applied to identify your risk may, with the support of databases: Pubmed Compounds, Chem Pubmed, Science Direct, IARC -International Agency of Research on Cancer- and Leaves of Security -MSDS-. Main results: 70 organic compounds, among which are some alkyl phenols, some of which pose a risk to human health and aquatic ecosystems, others are without risk characterization and a last group without information were identified. Conclusions: It is a pioneering work to understand the risks of urban, agricultural and industrial pollution of the river, passing through the city of Cali and will be useful for the control entities in the region, such as the Regional Autonomous Corporation of Valley Cauca, CVC, may take the measures necessary for the proper management of the river.
\end{abstract}

Key words: pollution, chromatography, alkyl phenols, environmental risks, Cauca River, Colombia.

\section{INTRODUCCIÓN}

Las aguas del río Cauca, a su paso por la zona urbana de la ciudad de Cali, presentan un alto grado de contaminación evidenciado en los siguientes hallazgos: Mutagenicidad, metales pesados $(\mathrm{Hg}, \mathrm{Cr}, \mathrm{Cd}$ y $\mathrm{Pb}$ ); pesticidas (diazinon, lindano, clordano, clorpirifos, etil-paration, malation, heptacloro aldrin, endosulfan), y de compuestos orgánicos entre los que destacan los alquilfenoles; riesgo de disrupción endocrina en mamíferos incluyendo el hombre (LARMAT et al., 2009; JIMÉNEZBOTERO et al., 2013; JIMÉNEZ-BOTERO \& SOTO-DUQUE, 2014).

El área de estudio está delimitada por el tramo del río Cauca localizado entre el puente de El Hormiguero y la Desembocadura del río Cali (Figura 1). El puente de El Hormiguero se denominó como estación de muestreo (E1), cuyas coordenadas geográficas son $03^{\circ} 18^{\prime} 09,2^{\prime \prime} \mathrm{N}$, longitud oeste $76^{\circ} 28^{\prime} 50,8^{\prime \prime} \mathrm{W}$, y altitud $978,3 \mathrm{msnm}$. La estación (E2): Desembocadura del Canal CVC-Sur 0322'40,2” N - 76²8'17,7” W, y altitud 967,1 msnm. La estación (E3): Bocatoma de la PTAP de Puerto Mallarino, 03²6'45,4" N - 76²8'30,4” W, y altitud 966,6 msnm. La estación (E4): Puente de Juanchito, 03026'59" N - 76²28'32,1" W, y altitud 963,6 msnm; y la estación (E5): Desembocadura del río Cali con coordenadas 03³0'20,9” N - 76²0'29,8” W, y altitud 965,8 msnm. 


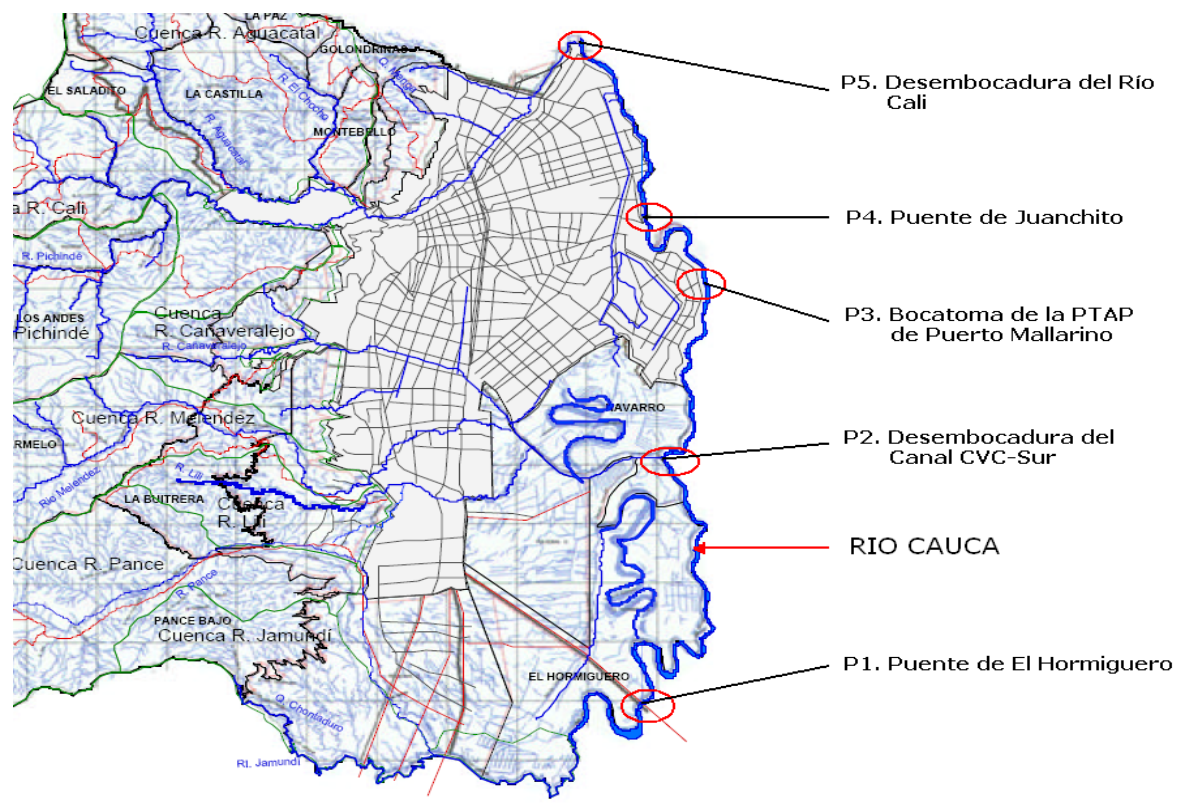

Figura 1. Zona de estudio. Ciudad de Cali indicando las estaciones de muestreo sobre el río Cauca, Colombia. Fuente: Cali en Cifras (2012, p 162)

El objetivo del presente trabajo fue el de analizar el riesgo ambiental en cinco estaciones de muestreo, en la zona de estudio (Figura 1), debido a la presencia de fenoles en el río Cauca a su paso por la zona urbana de Cali.

\section{MATERIALES Y MÉTODOS}

Se colectaron las muestras de agua de cada una de las cinco estaciones de muestreo, en envases ámbar de 4 litros, y se refrigeraron a $4^{\circ} \mathrm{C}$ hasta su filtración con filtros de membrana de 0,45 um, y extracción en fase sólida, con cartuchos OASIS HLB, se aplicó método OASIS de extracción para fenoles.

Los extractos se leyeron en un GC/MS Agilent $6890 \mathrm{~N}$ con MS detector Agilent $5973 \mathrm{~N}$ e inyección automática Agilent 7673. Columna capilar DB-5MS of $30 \mathrm{~m}$ de longitud, $250 \mu \mathrm{m}$ de diámetro y $0,30 \mu \mathrm{m}$ de espesor de película modelo No. J\&W 122-5532. Temperatura máxima de $325^{\circ} \mathrm{C}$. Flujo constante de $1 \mathrm{ml} / \mathrm{min}$. Detector de temperatura de $250^{\circ} \mathrm{C}$. Modo Pulsed Splitless, temperatura Quadropole de $150^{\circ} \mathrm{C}$. Fuente de ionización de $230^{\circ} \mathrm{C}$. Temperatura inicial del horno de $50^{\circ} \mathrm{C} / \mathrm{min}$, a una rata de $25^{\circ} \mathrm{C} / \mathrm{min}$ hasta $100^{\circ} \mathrm{C}$, luego a una rata de $5^{\circ} \mathrm{C} /$ min hasta $300^{\circ} \mathrm{C}$ durante 5 min. 


\section{RESULTADOS Y ANÁLISIS}

Usando la metodología anterior se detectaron en la Estación 1, los fenoles: Benzenemethanol,4-hydroxy, y Butylated hydroxytoluene - BHT (Figura 2).
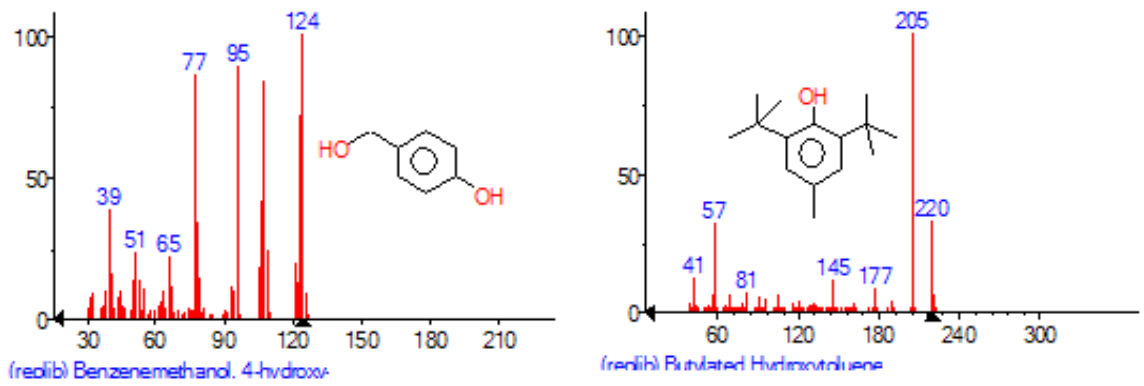

Figura 2. Cromatogramas de los fenoles: Benzenemethanol,4-hydroxy, y Butylated hydroxytoluene - BHT.

EL Benzenemethanol,4-hydroxy, presenta toxicidad aguda para la salud humana, y toxicidad en peces: LC-50 - Jordanella floridae - 10,5 mg/l - $2 \mathrm{~h}$; y el Butylated hydroxytoluene - BHT, registra toxicidad en peces: LC-50 - Oryzias sp. - 5,3 mg/l 48 h, en Daphnia y otros invertebrados acuáticos: EC50 - Daphnia pulex (Waterflea) - 1,44 mg/l - 48 h. KOUKI-INAI et al. (1988), registran el BHT como un compuesto que produce tumores en el hígado del ratón macho B6CC3F1, en el mismo sentido, CHIHOUNG et al. (1992), confirmaron que el BHT es un antioxidante para grasas y aceites, pero no presenta actividad antimutágenica, contrariamente aumenta la respuesta del test de Ames, en presencia de activador enzimático.

En la Estación 2, se identificaron los fenoles Phenol, 4-(1,1,3,3-tetramethylbutyl)-, y Phenol,2-methyl-4-(1,1,3,3-tetramethylbutyl)- (Figura 3).

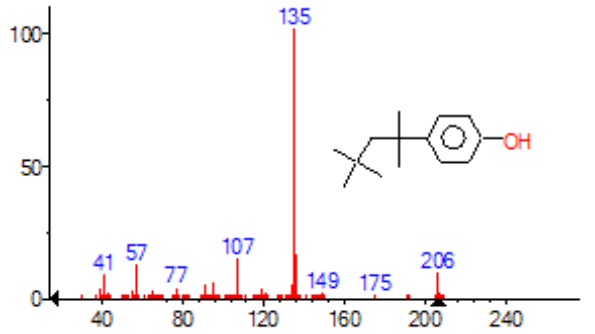

(mainlib) Phend, 441,1,3,3-tetramethylbutyl-

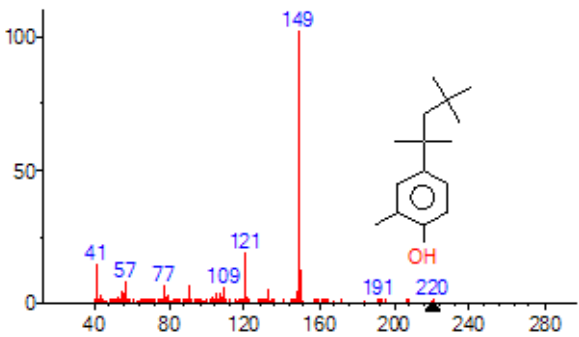

(mainlib) Phenol, 2-methi-4-11,1,3,3-tetramethybutvi)-

Figura 3. Cromatogramas de los fenoles: Phenol, 4-(1,1,3,3-tetramethylbutyl)-, y Phenol,2methyl-4-(1,1,3,3-tetramethylbutyl)-. 
El Phenol, 4-(1,1,3,3-tetramethylbutyl)- registra toxicidad acuática aguda y crónica (Categoría 1), puede formarse como un producto de degradación de los tensoactivos de alquilfenol en las aguas residuales, posee baja movilidad en el suelo, y en sedimentos (NCBI, 2005). Dado que su valor del Factor de Bioconcentración $\mathrm{BCF}$, es estimado en 6000, se espera que este compuesto se bio-acumule en los organismos acuáticos (TOXNET HOME-HSDB, 2012). También es reconocido disruptor endocrino, por lo anterior, puede acelerar la proliferación de células de cáncer en los organismos que entren en contacto con este compuesto.

En el mismo sentido, el Phenol,2-methyl-4-(1,1,3,3-tetramethylbutyl)-. De acuerdo a la ficha de seguridad MSDS de SIGMA-ALDRICH (2012), presenta toxicidad acuática crónica (Categoría 4), lesiones oculares graves (Categoría 1), irritaciones cutáneas (Categoría 2). La base de datos NCBI PUBCHEM COMPOUND (2005), no registra información toxicológica de este compuesto.

En la Estación 3 se encontraron: Butylated hydroxytoluene - BHT, Salicyl Alcohol, y el Phen-1,4-diol, 2,3-dimethyl-5-trifluoromethyl (Figura 4).

En esta estación reaparece el fenol Butylated hydroxytoluene - BHT, el cual estaba presente en la Estación 1; el Salicyl Alcohol registra irritaciones cutáneas y oculares (Categoría 2), toxicidad específica en determinados órganos - exposición única (Categoría 3); y el Phen-1,4-diol, 2,3-dimethyl-5-trifluoromethyl, no registra información de toxicidad y bio-actividad en la base de datos PubChem Compound.
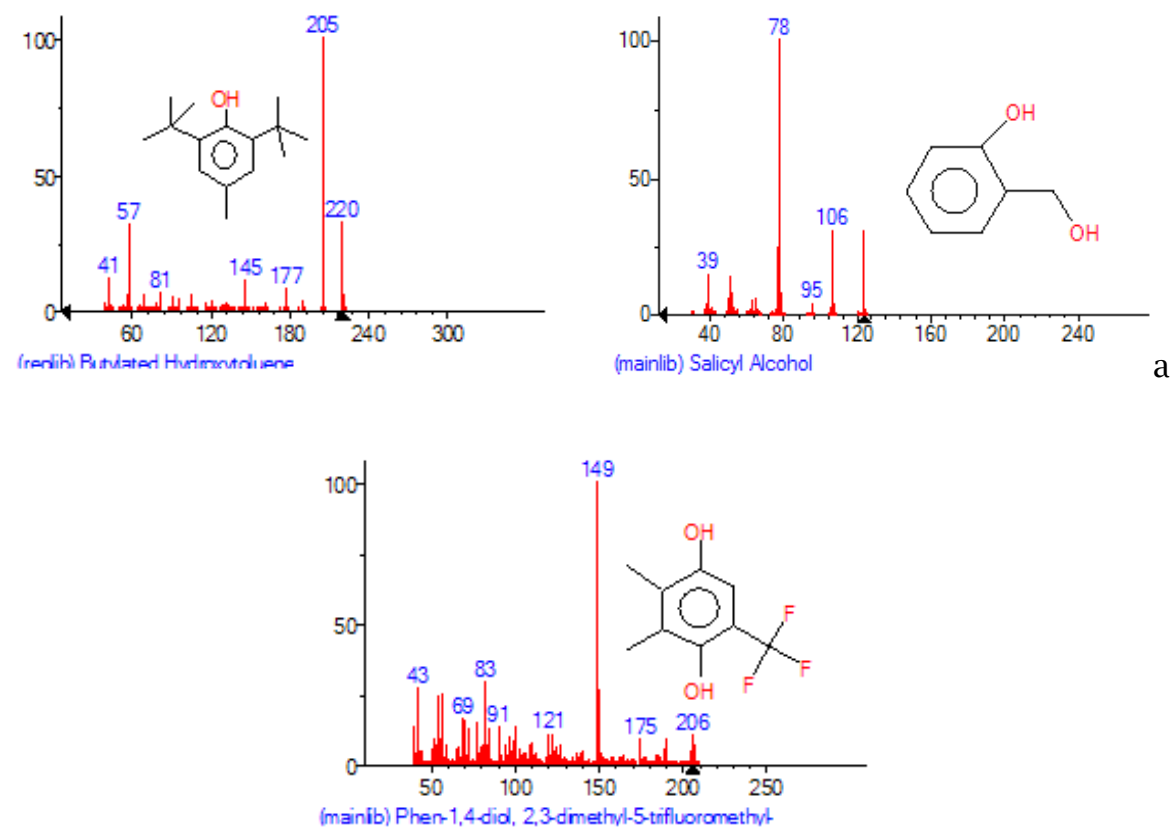

Figura 4. Cromatogramas de los fenoles: Butylated hydroxytoluene - BHT, Salicyl Alcohol, y el Phen-1,4-diol, 2,3-dimethyl-5-trifluoromethyl. 
La Estación 4 no registró presencia de fenoles. Contrariamente, en la Estación 5 se registraron los siguientes fenoles: Salicyl Alcohol, el cual se identificó en la Estación 3, y el Benzenemethanol,4-hydroxy, encontrado en la Estación 1.

\section{CONCLUSIONES}

Los alquifenoles son reconocidos disruptores endocrinos y promotores de la formación de células cancerígenas. En las cinco estaciones de muestreo se encontraron siete compuestos de esta familia, algunos de ellos se repiten en más de una estación.

Algunos de estos compuestos pueden formarse como un producto de degradación de los tensoactivos de alquilfenol en aguas residuales. Debido a sus propiedades, como movilidad en el suelo y en sedimentos, pueden bioconcentrarse en organismos acuáticos. Lo anterior, evidencia el riesgo ambiental presente en la zona de estudio.

En la Estación 4 no se registraron fenoles, mientras que en la Estación 3 se identificaron tres de estos compuestos; y en la Estación 2 se registró el más tóxico, correspondiente al Phenol, 4-(1,1,3,3-tetramethylbutyl)-, compuesto que registra toxicidad acuática aguda y crónica (Categoría 1).

\section{BIBLIOGRAFÍA}

CALI EN CIFRAS. 2012.- Alcaldía Santiago de Cali. Departamento Administrativo de Planeación. Cali (Colombia), $221 \mathrm{p}$.

CHIHOUNG, C., PEARSON, A.M. \& GRAY, J.I., 1992.- Effects of synthetic antioxidants (BHA, BHT and PG) on the mutagenicity of IQ-like compounds. Food Chemistry, 43: 177-183.

JIMÉNEZ-BOTERO, G.A. \& SOTO-DUQUE, A., 2014.- Análisis del riesgo ambiental potencial por compuestos orgánicos presentes en aguas del río Cauca, a su paso por la zona urbana de la ciudad de Cali (Colombia): Tesis de Magister en Desarrollo Sostenible y Medio Ambiente, Universidad de Manizales, Facultad de Ciencias Contables, Económicas y Administrativas.

JIMÉNEZ-BOTERO, G.A., SOTO-DUQUE, A. \& ÁLVAREZ-LEÓN, R., 2013.-Análisis del riesgo ambiental por alquil-fenoles presentes en aguas del río Cauca a su paso por la zona urbana de la ciudad de Cali (en) Memorias de Resúmenes del XLVIII Congreso Nacional de Ciencias Biológicas, Bogotá D.C., 6 a 9 de octubre, 2013 (resumen, p. 340). Rev. Asoc. Colomb. de Ciencias Biológicas, 25 (Supl. 01): 1-519.

KOUKI-INAI, T., KOBUKE, S., NAMBU, T., TAKEMOTO, E., KOU, H., NISHINA, M.F. et al., 1988.- Hepatocellular tumorigenicity of butylated hydroxytoluene administered orally to B6C3F1 mice. Cancer Science, 79: 49-58.

LARMAT, F.E., SOTO-DUQUE, A. \& BRAVO, E., 2009.- Evaluación de la actividad mutagénica del río Cauca en el área urbana de la ciudad de Cali - Colombia, por medio del test de Ames. Informe a COLCIENCIAS / Universidad del Valle / Universidad Autónoma de Occidente. Cali (Valle).

NCBI PUBCHEM COMPOUND., 2005.- Phenol,2-methyl-4-(1,1,3,3-tetramethylbutyl)- Compound Summary (CID 75206). National Center for Biotechnology Information, U.S. National Library of Medicine 8600 Rockville Pike, Bethesda MD, 20894, USA.

SIGMA-ALDRICH., 2012.- Material Safety Data Sheet. Product name: 4-(1,1,3,3-Tetrametilbutil)-o-cresol. Version 5.0.

TOXNET HOME-HSDB., 2012.- 4-(1,1,3,3-Tetramethylbutyl) PHENOL CASRN: 140-66-9 National Center for Biotechnology Information, U.S. National Library of Medicine 8600 Rockville Pike, Bethesda MD, 20894 USA. Disponible en: http://toxnet.nlm.nih.gov/cgi-bin/sis/search/r?dbs+hsdb:@term+@rn+@ rel+140-66- 